\title{
Catalyst Proximity-Induced Functionalization of h-BN with Quat Derivatives
}

\author{
Adrian Hemmi, ${ }^{* \dagger, \bullet}$ Huanyao Cun, ${ }^{* \dagger, \dagger, \bullet}$ Gabriele Tocci, ${ }^{\S}$ Adrian Epprecht, ${ }^{\dagger}$ Bart Stel, ${ }^{\|, \perp}$ \\ Magalí Lingenfelder, $\|, \perp \odot$ Luis Henrique de Lima,,,$\nabla \odot$ Matthias Muntwiler, ${ }^{\circledR \odot}$ Jürg Osterwalder, ${ }^{\dagger}$ \\ Marcella Iannuzzi, ${ }^{\S}$ and Thomas Greber* ${ }^{* \dagger}$ \\ ${ }^{\dagger}$ Physik-Institut and ${ }^{\S}$ Department of Chemistry, Universität Zürich, 8057 Zürich, Switzerland \\ ${ }^{\ddagger}$ Institute of Bioengineering, "Max Planck-EPFL Laboratory for Molecular Nanoscience, and ${ }^{\perp}$ Institut de Physique, EPFL, 1015 \\ Lausanne, Switzerland \\ ${ }^{\#}$ Swiss Light Source, Paul Scherrer Institut, 5232 Villigen PSI, Switzerland \\ ${ }^{\nabla}$ Centro de Ciências Naturais e Humanas, Universidade Federal do ABC, 09210-580, Santo André, Brazil
}

Supporting Information

ABSTRACT: Inert single-layer boron nitride (h-BN) grown on a catalytic metal may be functionalized with quaternary ammonium compounds (quats) that are widely used as nonreactive electrolytes. We observe that the quat treatment, which facilitates the electrochemical transfer of two-dimensional materials, involves a decomposition of quat ions and leads to covalently bound quat derivatives on top of the $2 \mathrm{D}$ layer. Applying tetraoctylammonium and $\mathrm{h}-\mathrm{BN}$ on rhodium, the reaction product is top-alkylized $\mathrm{h}-\mathrm{BN}$ as identified with high-resolution

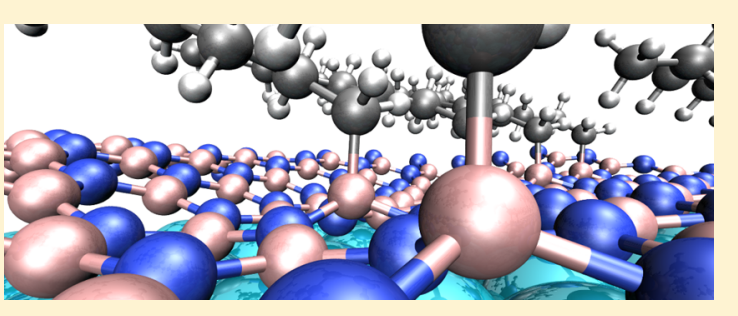
$\mathrm{X}$-ray photoelectron spectroscopy. The alkyl chains are homogeneously distributed across the surface, and the properties thereof are well-tunable by the choice of different quats. The functionalization further weakens the $2 \mathrm{D}$ material-substrate interaction and promotes easy transfer. Therefore, the functionalization scheme that is presented enables the design of $2 \mathrm{D}$ materials with tailored properties and with the freedom to position and orient them as required. The mechanism of this functionalization route is investigated with density functional theory calculations, and we identify the proximity of the catalytic metal substrate to alter the chemical reactivity of otherwise inert h-BN layers.

KEYWORDS: Hexagonal boron nitride, quaternary ammonium compounds, electrochemical process, 2D materials functionalization, $2 \mathrm{D}$ materials transfer, heterogeneous catalysis on metals

$\mathrm{E}$ nabling materials requires a set of tools and strategies to tune the properties of a desired compound for its final application. Because two-dimensional (2D) materials are expected to become building blocks for electronic devices beyond the silicon technology ${ }^{1,2}$ or to be used as membranes for efficient ion separation and DNA sequencing, ${ }^{3,4}$ a corresponding toolbox with knowledge about reactivities and functionalization pathways needs to be established. Although graphene-the single 2D layer of graphite-can rely on carbonbased organic chemistry, the appliances to enable hexagonal boron nitride (h-BN) are limited, ${ }^{5}$ and edge functionalization of the h-BN sheets is preferred. ${ }^{6,7}$ To date, solution-based hydroxylation or alkylation of h-BN requires radical chemistry at elevated temperatures or long processing times and the application of highly reactive agents, such as peroxides, ${ }^{8}$ dibromocarbene, ${ }^{9}$ and naphthalide. ${ }^{10}$ Our work advances the radical h-BN alkylation route but employs a single reaction step at room temperature with chemicals that are easy to handle and store and a simple solvent purge for product cleaning.

The functionalization is realized with a quaternary ammonium compound (quat)/acetonitrile solution in an electrochemical cell, which is operated below the electrochemical window of quats (i.e., a voltage at which the molecules are reduced). With tetraoctylammonium (TOA) as the quat, we achieve the alkylation of single-layer h-BN on $\mathrm{Rh}(111)$. The alkylation reaction is assigned to a side reaction of an electrochemical quat reduction under aprotic conditions. $^{11} \mathrm{Ab}$ initio density functional theory (DFT) calculations identify the key ingredient for this simple alkylation scheme to be the transition-metal substrate that substantially alters the chemical reactivity on the side of h-BN farther away from the metal. ${ }^{12}$ The functionalization process features self-termination as observed by scanning probe microscopy. The alkylation further occurs on only one side of h-BN, as measured by angle-dependent X-ray photoelectron spectroscopy (XPS), and the functionalizations sustain extreme conditions, as demonstrated by annealing to $830{ }^{\circ} \mathrm{C}$.

Quaternary ammonium cations are widely used as electrolytes in batteries or capacitors because of their electrochemical

Received: May 1, 2019

Revised: $\quad$ August 7, 2019

Published: August 13, 2019 

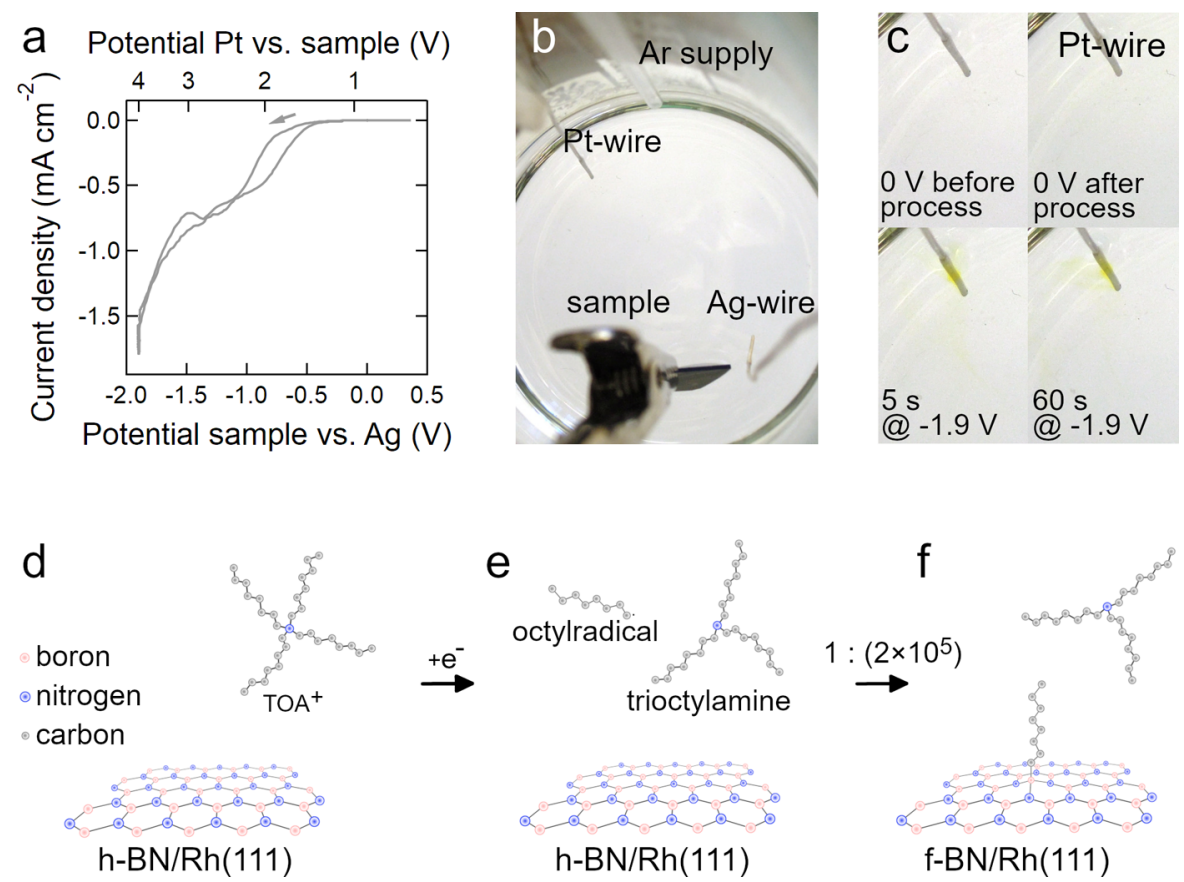

Figure 1. Electrochemical reactions in the $0.1 \mathrm{M}$ TOABr/acetonitrile solution with a h-BN/Rh(111) cathode and a Pt anode. (a) Voltammogram during the $\mathrm{TOABr}$ process with the potential between the sample and reference electrode as well as the potential between the Pt counter electrode and sample. (b) Top view of the three-electrode setup in an argon atmosphere and (c) pictures of the Pt wire at different times during the TOA process. (d, e) Schematics of the TOA decomposition reaction. A small number of the alkyl radicals in (e) are able to covalently bind to h-BN on $\mathrm{Rh}(111)$ to form functionalized boron nitride (f-BN) (f).

stability. ${ }^{11,13}$ Electrochemistry with quats started to impact the enabling of $2 \mathrm{D}$ materials when they were applied to the activation of graphene to promote carboxylation ${ }^{14}$ and to facilitate the exfoliation of graphene from transition metals. ${ }^{15}$ The latter concept of facile transfer has inspired a wide range of research activities, and it was successfully applied with different quats ${ }^{16}$ to a variety of $2 \mathrm{D}$ material systems. ${ }^{15-19}$ However, these electrochemical processes were assigned to involve quat intercalation. Here, the data unambiguously exclude intercalation and suggest a covalent functionalization with quat derivatives instead. Nevertheless, quat-induced delamination is the prime feature of the functionalization route presented and gives access to tailored $2 \mathrm{D}$ building blocks. The simplicity of the chemicals involved provides the opportunity for easy customization of the functional groups, the self-terminating process ensures a homogeneous distribution and the covalent bond grants persistence and reliability. But it is the easy transferability which enables the placement of such a customized $2 \mathrm{D}$ material where needed and with the functionalization pointing in the direction for which it is intended (e.g., it allows us to design membranes with two different faces such as the ones used for ion transport in aqueous $\mathrm{KCl}$ solutions, ${ }^{18}$ which exhibit asymmetric ion transport behavior).

The alkyl-functionalized h-BN on $\mathrm{Rh}(111)$ features a distinct elemental composition, annealing behavior, and electronic valence structure as compared to pristine h-BN. Hence, we use nomenclature with the prefix "functionalized" (f) (i.e., functionalized boron nitride $(\mathrm{f}-\mathrm{BN})$ ).

\section{RESULTS AND DISCUSSION}

To understand the nature of the TOA-treatment effects on a 2D layer/metal surface, the electrochemistry of this procedure is first examined in detail. Next, synchrotron-based high- resolution XPS measurements rationalize the structure of TOABr-treated h-BN/Rh(111). Finally, a series of consecutive TOA treatments confirm that the TOABr process leads to functionalized $\mathrm{BN}$ (f-BN).

For the $\mathrm{TOABr}$ process, a three-electrode setup is employed, with a $\mathrm{Pt}$ wire as a counter electrode, a $\mathrm{Ag} / \mathrm{AgBr}$ pseudoreference electrode (i.e., a $\mathrm{Ag}$ wire immersed in $\mathrm{TOABr} /$ acetonitrile $^{15}$ ), and the $\mathrm{h}-\mathrm{BN} / \mathrm{Rh}(111)$ thin film sample as the working electrode. The top view of the setup is shown in Figure $1 \mathrm{~b}$. The process requires a sample voltage of $-1.9 \mathrm{~V}$ vs $\mathrm{Ag} / \mathrm{AgBr}$ for $10 \mathrm{~min}$, followed by $-0.2 \mathrm{~V}$ for $20 \mathrm{~s}$. The current at $-1.9 \mathrm{~V}$ vs $\mathrm{Ag} / \mathrm{AgBr}$ is constant and on the order of $\sim 1.5 \mathrm{~mA} / \mathrm{cm}^{2} \cdot{ }^{14,16,17}$ It was suggested that the constant current is caused by a continuous double-layer charging due to continuous $\mathrm{TOA}^{+}$intercalation, which is solely a polarization process. ${ }^{16}$ From an electronic perspective, such a process corresponds to the charging of a capacitor, where no charges are exchanged between electrodes and electrolyte. Figure 1a displays the voltammogram of the complete process, and an exponential current increase below $-1.5 \mathrm{~V}$ vs $\mathrm{Ag} / \mathrm{AgBr}$ points toward an electrochemical reaction. Figure 1c displays several snapshots of the $\mathrm{Pt}$ counter electrode before, during, and after the $\mathrm{TOABr}$ process. At $-1.9 \mathrm{~V}$ vs $\mathrm{Ag} / \mathrm{AgBr}$, a yellow color emerges in the solution around the $\mathrm{Pt}$ wire (Figure $1 \mathrm{~b}$ ), which is examined in detail in Figure $\mathrm{S} 1$ of the Supporting Information (SI). The temporal coincidence of the large process current with this localized yellow coloring indicates an oxidation reaction at the positive Pt electrode. Considering the oxidation products of all chemical species in the solution suggests that the color is bromine-based, and we assigned it to the bromine reaction:

$2 \mathrm{Br}^{-} \rightarrow \mathrm{Br}_{2}+2 \mathrm{e}^{-}$ 

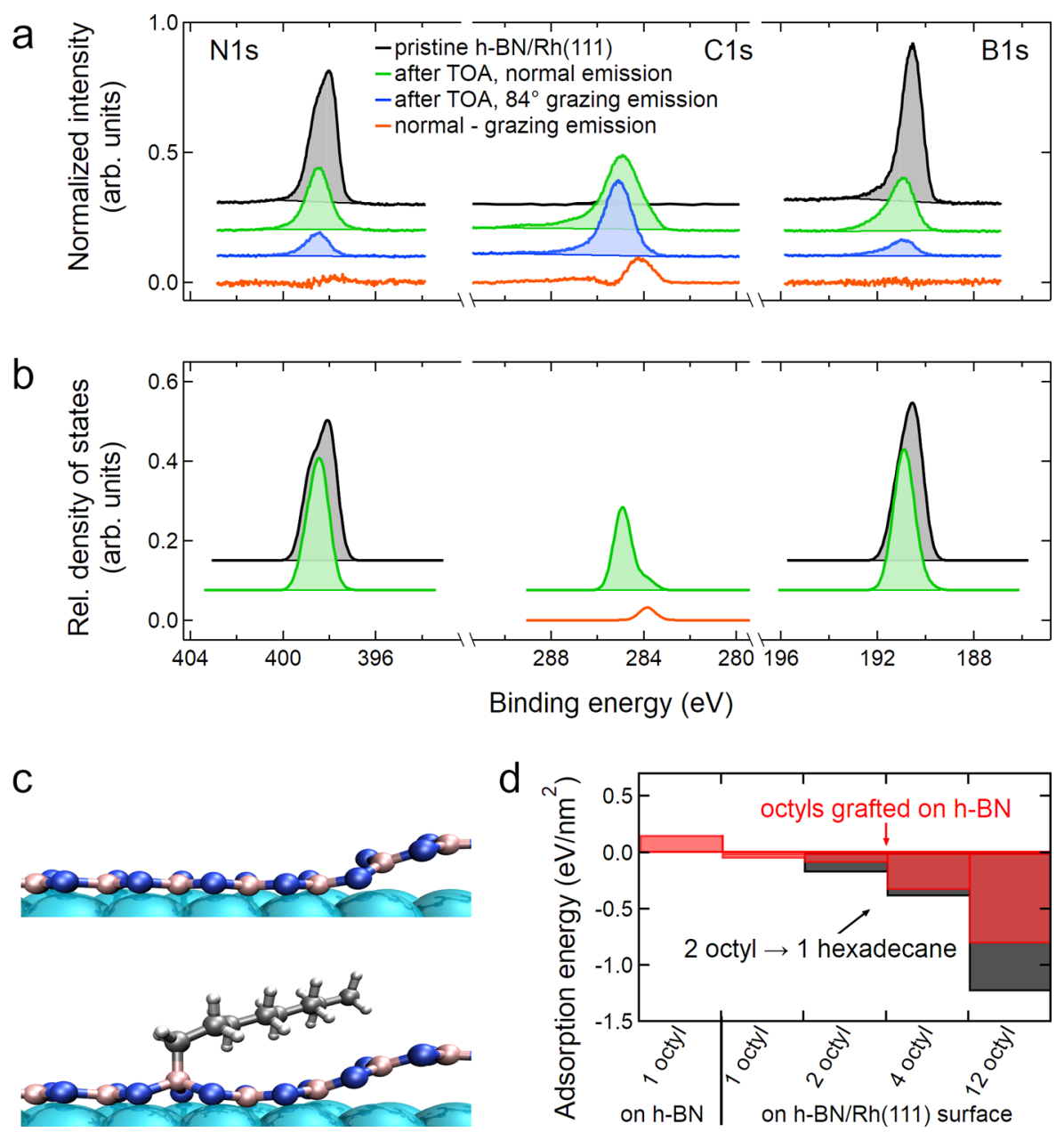

Figure 2. Measured and simulated XPS spectra of $\mathrm{h}-\mathrm{BN} / \mathrm{Rh}(111)$ before and after TOA treatment. (a) XPS data of pristine material (black), after TOA treatment at normal emission (green), at an $84^{\circ}$ grazing angle (blue), and the difference between the last two (orange). The photon energy is $850 \mathrm{eV}$, and all signals are normalized with their corresponding photoemission cross sections. The grazing angle data are scaled with a factor of 5.7 such that the carbon peak heights coincide. (b) Calculated XPS signals from the energy eigenvalues of the h-BN/Rh(111) unit cell with and without 12 octyl radicals which were relaxed above h-BN/Rh(111). (c) Cross-sectional view of the structures from the XPS calculations. For clarity, only one octyl is shown. (d) DFT total energy differences of octyl radical adsorption on free-standing h-BN and h-BN/Rh(111).

With an oxidation reaction at the $\mathrm{Pt}$ counter electrode, charge neutrality in the solution can be sustained only with a reduction reaction at the sample electrode. Therefore, the TOA process current is assigned to a chemical reaction and not cation intercalation/solution polarization, although that could still occur. Because the $\mathrm{Pt}$ sample voltage exceeds the electrochemical window of quaternary ammonium cations, which is about $4 \mathrm{~V}$ (Figure $1 \mathrm{a}$ ), ${ }^{20,21} \mathrm{a} \mathrm{TOA}^{+}$decomposition reaction most likely occurs on the h-BN/ $\mathrm{Rh}(111)$ surface. In anhydrous and aprotic solvents, the tetraalkylammonium reduction is known to form trialkylamine and octyl radicals. ${ }^{11}$ This reaction is schematically drawn in Figure $1 \mathrm{~d}, \mathrm{e}$ and is electrode-surface-independent per se. For the presently used h$\mathrm{BN} / \mathrm{Rh}(111)$ electrodes, DFT calculations predict a chemisorbed octyl chain from a reduced and dissociated $\mathrm{TOA}^{+}$to be $1.6 \mathrm{eV}$ more stable than the intact state. Chemisorption occurs at boron sites close to the $\mathrm{Rh}(111)$ surface and leads to functionalization as depicted in Figure if (further details in the SI).

For the structure determination of TOABr-processed h-BN/ $\mathrm{Rh}(111)$, the sample is thoroughly rinsed with acetonitrile (section 2 in Supporting Information), and high-resolution XPS is employed. Figure 2a displays XPS data of pristine, in- situ-prepared h-BN on $\mathrm{Rh}(111)$ (black) and of the TOABrtreated $\mathrm{h}-\mathrm{BN} / \mathrm{Rh}(111)$ surface at normal (green) and at grazing angle emission (blue). The pristine h-BN/Rh(111) sample features two nitrogen components, ${ }^{22}$ which relate to an intrinsic property of the $\mathrm{h}-\mathrm{BN} / \mathrm{Rh}(111)$ surface that is a real space corrugation of the adlayer, the so-called "nanomesh". ${ }^{23}$ After the TOA treatment, this double-peak characteristics is lost. Such a loss of electronic structure has been reported to occur after $\operatorname{tin}^{24}$ or hydrogen intercalation, where it coincided with a flattening of the physical corrugation of the nanomesh. ${ }^{25}$ Comparing XPS intensities, we find that the boron and nitrogen signals undergo an attenuation of about 50\% upon TOA treatment. Because the TOA treatment allows the transfer of $95 \% \mathrm{~h}-\mathrm{BN}$ from $\mathrm{Rh}(111)^{18}$ and because the grazing angle data indicate a top carbon cover, the boron and nitrogen signal reduction is assigned to the attenuation through a carbon layer due to the nanometer short inelastic electron mean free path of the photoelectrons. Following the same inelastic electron mean free path argument, the comparison of grazing angle with normal emission data allows us to discriminate species of the same element at different depths below the surface. The differences in these signals are displayed (orange) and reveal a single boron-nitrogen layer and two 
different carbon species. The single nitrogen species indicates that traces of nitrogen from either TOA or acetonitrile are not detected. From the XPS data, a carbon quantity is determined with a 1.0 monolayer reference $\left(3.7 \times 10^{15}\right.$ atoms $\left./ \mathrm{cm}^{2}\right)$ for the pristine h-BN layer (black). This yields a total carbon quantity of $45 \%$ monolayer and $7.4 \%$ monolayer for the lower-bindingenergy species. The estimated coverage represents an upper limit of carbon that originates from the TOA process because the surface was exposed between rinsing and the XPS measurement to ambient conditions, from where it may pick up additional carbon.

For comparison with the experiment, XPS spectra of pristine h-BN/ $\mathrm{Rh}(111)$ and of a system with 12 octyls covalently bonded to $\mathrm{B}$ atoms in the pore of a single nanomesh unit cell were calculated. In Figure 2c, we show the DFT structures of cross sections across the pristine $\mathrm{h}-\mathrm{BN} / \mathrm{Rh}(111)$ and the $\mathrm{f}$ $\mathrm{BN} / \mathrm{Rh}(111)$. The coordinates of the energy-optimized systems are provided in the SI. The TOA derivatives prefer the proximity to the $\mathrm{Rh}$ substrate and bind the strongest in locations where the h-BN layer is close to the metal catalyst. The calculations of the XPS core-level energies are based on the half-core hole approximation, where initial and final state effects are accounted for by electronic energy eigenvalue calculations after removing half an electron from the core state. $^{26}$ The core-state energy values thus obtained are convoluted with a Gaussian function with a full width at half-maximum (fwhm) of $0.82 \mathrm{eV}$ and displayed in Figure $2 \mathrm{~b}$. On absolute binding energy scales, all simulated values are shifted to match the experimental results, and only peak-shape changes are discussed. The $\mathrm{N} 1 \mathrm{~s}$ core-level splitting of the pristine $\mathrm{h}-\mathrm{BN} / \mathrm{Rh}(111)$ nanomesh and its loss after the octyl radical adsorption are well reproduced. The shoulder at lower binding energy of the $\mathrm{C}$ 1s spectrum (highlighted by the bottom orange line in the middle panel of Figure $2 b$ ) represents a second carbon species spatially closer to h-BN and directly bound to boron. The covalent character of the carbon-boron bond is inferred from the "pop out" of the involved boron atom off the h-BN plane by $0.6 \AA$ and indicates boron with an $\mathrm{sp}^{3}$ configuration (Figure $2 \mathrm{c}$ ). The agreement between the experimental and calculated XPS features suggests an $\mathrm{f}-\mathrm{BN}$ structure that is a monolayer h-BN with covalent octyl functionalization on top. Intercalation or bottom functionalization is not detected and has to be below the sensitivity of XPS. Complementary, angle-dependent XPS measurements and surface composition simulations, which do not rely on DFT, can be found in section 3 of the Supporting Information.

We performed a number of DFT structure relaxations of octyl radicals on freestanding $\mathrm{h}-\mathrm{BN}$ and on $\mathrm{h}-\mathrm{BN} / \mathrm{Rh}(111)$ and computed adsorption energies (more details in the SI). Adding more and more octyl radicals leads to two stable spacial arrangements, namely, chemisorbed octyl chains on specific boron sites in h-BN and the physisorption of dimerized octyl radicals (i.e., hexadecanes). An overview of the adsorption energies is provided in Figure $2 \mathrm{~d}$. The radical dimerization appears to be the energetically favored process, while the octyl chemisorption on metal-supported h-BN represents a side product of the electrochemical reaction. A comparison of the total charge flowing through the $\mathrm{h}-\mathrm{BN} / \mathrm{Rh}(111)$ electrode with the number of electrons used for TOA decomposition and chemisorption of $30 \%$ of a monolayer carbon allows an estimation of the octylradical-surface functionalization yield of about $1:\left(2 \times 10^{5}\right)$. Such a low alkylation yield confirms that the covalent functionalization is indeed a side reaction and the main reaction is assigned to radical dimerization in solution. These dimerization reactions finally explain the mismatch between the measured process current and the orders of magnitude lower surface modifications as reported earlier. $^{14,16,17}$

Because electrochemistry, XPS, and DFT resolve the structure of TOABr-treated h-BN/Rh(111), real-space imaging is used to confirm uniform modifications across the sample. Atomic force microscopy (AFM) images are displayed in Figure 3. The faint lines are single atomic $\mathrm{Rh}(111)$ steps. $^{27}$
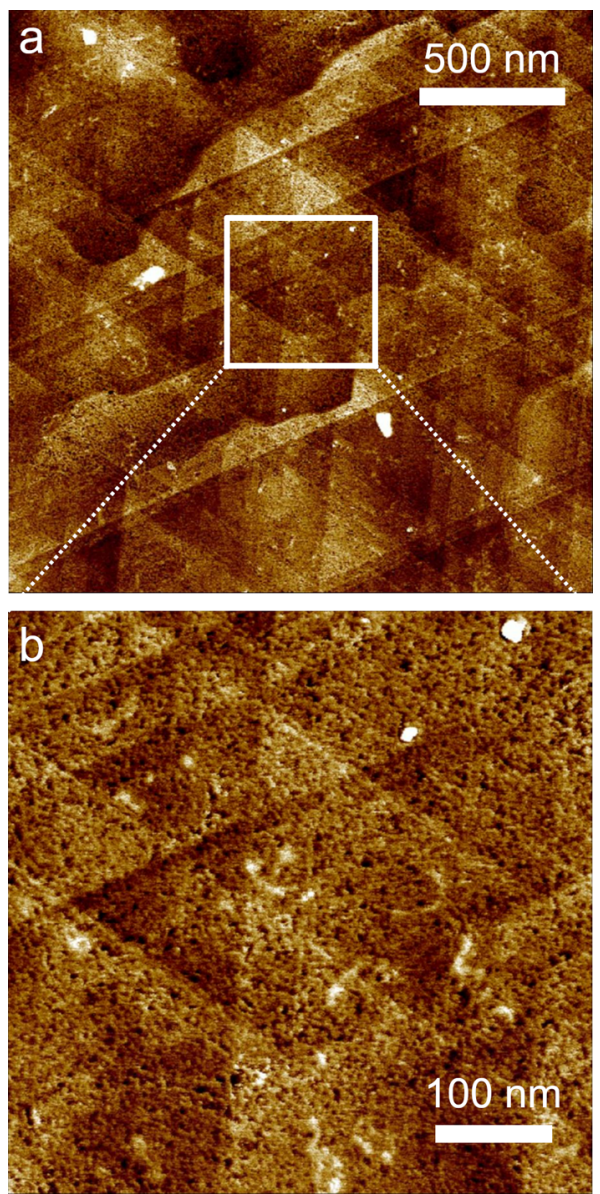

Figure 3. AFM images of TOA-treated h-BN/Rh(111) at room temperature. (a) $2 \times 2 \mu \mathrm{m}^{2}$ and (b) $500 \times 500 \mathrm{~nm}^{2}$ scan areas, which are measured using a PF-HIRS tip in Peak Force mapping mode. The three sets of faint lines rotated by $60^{\circ}$ with respect to each other are single atomic steps of the $\mathrm{Rh}(111)$ surface, which are typical of the single-crystalline $\mathrm{Rh}$ films. ${ }^{27}$

Therefore, the lawnlike surface morphology is atomically thin, and on the micrometer scale, these features homogeneously cover the surface. Clustering or island formation are not observed, suggesting that the surface functionalization reaction is a self-terminating process.

To further confirm that the TOABr process conditions lead to covalently attached carbon chains on the h-BN/ $\mathrm{Rh}(111)$ substrate, we performed a series of $\mathrm{TOABr}$ treatments and ambient condition exposures with elemental and electronic valence structure analysis. The experimental protocol is schematically displayed in Figure $4 \mathrm{~d}$ and starts with the annealing of $\mathrm{h}-\mathrm{BN} / \mathrm{Rh}(111)$ at ultrahigh vacuum (UHV) to $830{ }^{\circ} \mathrm{C}$, followed by XPS and normal emission ultraviolet 

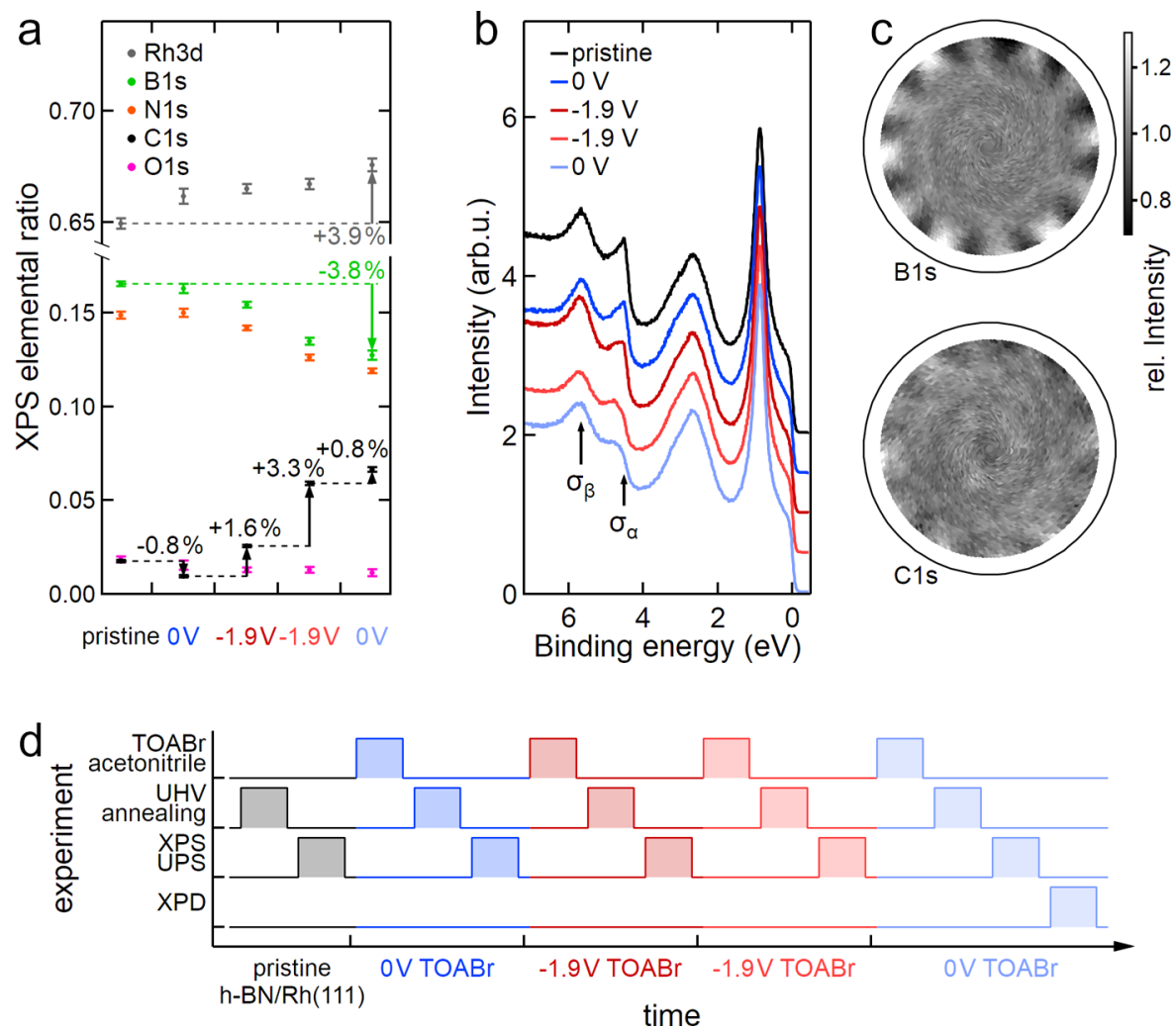

Figure 4. Elemental and electronic $\mathrm{h}-\mathrm{BN} / \mathrm{Rh}(111)$ changes after the $\mathrm{TOABr}$ process and annealing to $830{ }^{\circ} \mathrm{C}$. (a) Elemental ratios obtained from $\mathrm{Mg} \mathrm{K} \alpha$ normal emission XPS data and (b) He I $\alpha$ UPS of pristine h-BN/Rh(111) (black) and after a series of TOABr solution exposures, $0 \mathrm{~V}$ vs $\mathrm{Ag} / \mathrm{AgBr}$ (blue) and $-1.9 \mathrm{~V}$ vs $\mathrm{Ag} / \mathrm{AgBr}$ (red). (c) XPD patterns of $\mathrm{B} 1 \mathrm{~s}$ and $\mathrm{C} 1 \mathrm{~s}$ after the final TOABr immersion at $0 \mathrm{~V}$ vs $\mathrm{Ag} / \mathrm{AgBr}$. The XPD signals are averaged by the azimuthal mean at each polar angle, which enhances the forward scattering contrast at grazing angle and induces intensity values of around 1 . (d) Schematic diagram of the experimental protocol vs time.

photoelectron spectroscopy (UPS) measurements. Subsequently, the sample is cycled through a series of immersions into $0.1 \mathrm{M}$ TOABr for $10 \mathrm{~min}$, followed by acetonitrile rinsing, UHV annealing, and inspection with XPS and UPS. The only parameter varied in these cycles is the potentiostat voltage during the $\mathrm{TOABr}$ solution immersion, which is $0,-1.9,-1.9$, and $0 \mathrm{~V}$ vs $\mathrm{Ag} / \mathrm{AgBr}$.

Figure 4a summarizes the elemental composition from XPS of the h-BN/ $\mathrm{Rh}(111)$ surface as a function of the $\mathrm{TOABr}$ treatment and annealing iteration. As expected, the largest elemental fractions are rhodium, boron, and nitrogen. The rhodium contribution steadily increases, while boron and nitrogen decrease, which reflects a continuous loss of h-BN. After both $-1.9 \mathrm{~V}$ vs $\mathrm{Ag} / \mathrm{AgBr}$ treatments, the carbon quantity is more than doubled, which is not observed after the $0 \mathrm{~V}$ treatments. Inferring a 1.0 monolayer reference for the XPS signal of pristine h-BN, the carbon content increases after the $-1.9 \mathrm{~V}$ treatments correspond to 5 and $10 \%$ monolayers. Comparison with the carbon content directly after the TOA treatment (45\% monolayer, see Figure $2 \mathrm{a}$ ) indicates that the largest impact of UHV annealing is carbon desorption. To localize the remaining carbon, the valence states are examined with $\mathrm{He}$ I $\alpha$ UPS and shown in Figure $4 \mathrm{~b}$. The two peaks at the lowest binding energies reflect rhodium and the $\sigma_{\alpha}$ and $\sigma_{\beta}$ boron nitride bands, which correspond to the nanomesh wire and pore states, respectively. ${ }^{28}$ The $\sigma$ bands are sensitive to the adsorption of molecular species or intercalation, where the $\sigma_{\beta}$ band disappears. ${ }^{24,25}$ Here, every process step attenuates the intensity of both boron nitride bands, which is in line with the
XPS data in Figure 4a. The effect of the $-1.9 \mathrm{~V}$ TOABr treatments differs from the $0 \mathrm{~V}$ treatments by an extra broadening of the $\sigma_{\alpha}$ bands. The intact $\sigma_{\beta}$ band and a broadened $\sigma_{\alpha}$ band imply a h-BN/Rh(111) nanomesh structure with a modified wire region. Scanning tunneling microscopy (STM) measurements after the final annealing cycle complement these findings, and the pristine nanomesh structure is detected with additional nanometer-sized protrusions (Figure S5 of the Supporting Information). We assign these protrusions to covalently bound remains of the alkyl chains, which, based on the UPS data, persist only in the wire region of $\mathrm{h}$-BN. An additional effect is measured by X-ray photoelectron diffraction (XPD) after the final annealing cycle and displayed in Figure 4c. The six forward-scattering directions of boron are observed for carbon at the same polar and azimuthal positions. This indicates the integration of carbon into the h-BN layer, and the presence of graphene patches in the h-BN layer is confirmed by STM (Figure S5 in Supporting Information). Such lateral graphene-h-BN heterostructures were also observed after consecutive h-BN and graphene growth on transition metals. ${ }^{29}$ In the present case, the carbon precursor is provided via electrochemical alkylation prior to annealing.

\section{CONCLUSIONS}

The $\mathrm{TOABr}$ treatment for facile $2 \mathrm{D}$ layer delamination is an electrochemical reaction with bromide oxidation at the counter electrode and TOA reduction on the sample working electrode. DFT calculations suggest that the radicalic TOA 
decomposition product can covalently bind to single-layer h$\mathrm{BN}$ as long as the latter is in close proximity to $\mathrm{Rh}(111)$. The covalent functionalization is experimentally confirmed by a series of $\mathrm{TOABr}$ exposures and photoelectron spectroscopy investigations. We suggest that these TOABr-process effects are generally applicable to other systems of $2 \mathrm{D}$ layers in the vicinity of transition metals (e.g., graphene on iridium) and to other ammonium ions. Apart from easy-to-handle chemicals and processes, the advantage of this functionalization concept is threefold: (i) the functionalizations are homogeneously distributed across the surface in a self-terminating reaction, (ii) the chemically functionalized 2D layers become easily amenable to transfer via electrochemical delamination, and (iii) the functionalizations are covalently attached to the $2 \mathrm{D}$ layer, which makes such systems persistent to extreme conditions and is equivalent to a new material (i.e., f-BN). By exploiting these advantages, it is now possible to design atomically thin two-sided membranes with two chemically different faces.

\section{METHODS}

The h-BN monolayer is prepared on 4 in. $\mathrm{Rh}(111)$ thin-film wafers with the procedures and facility described by Hemmi et al. ${ }^{27}$ The electrochemical alkylation is achieved in Ar degassed, dehydrated acetonitrile and a home-build potentiostat employing a Keithley 2602B source measure unit and a National Instruments USB-6351 card to measure the reference electrode potential. Chemicals are purchased from Fluke. The sample cleaning procedure after the $\mathrm{TOABr}$ /acetonitrile treatment and before any other measurement is described in detail in section 2 of the Supporting Information.

The standard XPS, XPD, and UPS measurements were carried out in an ESCALAB 220, ${ }^{30}$ and the high-resolution XPS data were recorded in the photoemission and atomic resolution laboratory (PEARL) synchrotron beamline at the Swiss Light Source (SLS). ${ }^{31}$ AFM data are acquired with PFHIRS tips in Peak Force tapping mode (Bruker Dimension FastScan). The STM experiments were performed in a variable-temperature scanning tunneling microscope (Omicron VT-STM).

DFT simulations have been performed with the CP2K code $^{32}$ using the PBE-rVV10 density functional. ${ }^{33-35}$ The ground-state calculations have been carried out with the Gaussian plane wave method, the molecular orbitals of the valence electrons are expanded into a combination of Gaussian and plane waves, and the core electrons are treated using Goedecker-Teter-Hutter pseudopotentials. ${ }^{36}$ For the Gaussian basis set expansion, the valence orbitals have been expanded into molecularly optimized DZVP basis sets for all elements except $\mathrm{Rh}$, where orbitals were expanded using a molecularly optimized SZVP basis. ${ }^{37}$ For the auxiliary plane wave expansion, a cutoff of $600 \mathrm{Ry}$ has been used. The calculation of the N, C, and B K-edges to reproduce the XPS data has been achieved by applying the Gaussian augmented plane wave method under the half-core hole approximation, ${ }^{38}$ where for the light elements $(\mathrm{N}, \mathrm{B}, \mathrm{C}$, and $\mathrm{H})$ all electrons are explicitly considered (no pseudopotentials). The 6-311G** allelectron basis sets were employed to expand the molecular orbitals of the light elements in the Gaussian augmented plane wave method for the K-edge calculations.

\section{ASSOCIATED CONTENT}

\section{Supporting Information}

The Supporting Information is available free of charge on the ACS Publications website at DOI: 10.1021/acs.nanolett.9b01792.

Additional optical data and its evaluation of the bromide oxidation reaction during the $\mathrm{TOABr}$ process, information about sample cleaning and cleanliness after $\mathrm{TOABr}$ /acetonitrile solution exposures, angle-dependent XPS of the TOABr treated h-BN/Rh(111) surface, monochromatic $\mathrm{Al} \mathrm{K} \alpha$ XPS data confirming the carbon poisoning of h-BN after the TOABr treatments, STM data of TOABr-treated and annealed h-BN/Rh(111), and coordinates of the intact TOA and dissociated TOA on $\mathrm{h}-\mathrm{BN} / \mathrm{Rh}(111)$ as well as of the 0.28 monolayer of grafted octyls, obtained from DFT structure relaxations (PDF)

Coordinate files of the nanomeshes (ZIP)

\section{AUTHOR INFORMATION}

\section{Corresponding Authors}

*E-mail: hemmi@physik.uzh.ch. Phone: +41 446355744.

*E-mail: hycun1@physik.uzh.ch. Phone: +41 446355744.

*E-mail: greber@physik.uzh.ch. Phone: +41 446355744.

ORCID

Magalí Lingenfelder: 0000-0003-1362-8879

Luis Henrique de Lima: 0000-0001-8432-2637

Matthias Muntwiler: 0000-0002-6628-3977

Jürg Osterwalder: 0000-0001-9517-641X

Marcella Iannuzzi: 0000-0001-9717-2527

Thomas Greber: 0000-0002-5234-1937

\section{Author Contributions}

-These authors contributed equally to this work

Notes

The authors declare no competing financial interest.

\section{ACKNOWLEDGMENTS}

Financial support by the European Commission under Graphene Flagship Core 2, no. 785219, SNF Sinergia grant CRSI22_122703 "Boron nitride nanomesh as a template for guided self-assembly of molecular arrays", the Research Priority Program (URPP) for solar light to chemical energy conversion (LightChEC) of the University of Zurich, and the SNF Ambizione grant "Ab Initio Nanofluidics: Electronic Structure and Transport Properties for Osmotic Energy Conversion" is gratefully acknowledged. High-resolution XPS was performed at the PEARL beamline of the Swiss Light Source, Paul Scherrer Institut (PSI), Villigen, Switzerland. The authors are grateful for the generous allocation of computing resources from the Swiss National Supercomputing Center (CSCS) under project IDs s657 and uzh1.

\section{REFERENCES}

(1) Novoselov, K. S.; Geim, A. K.; Morozov, S. V.; Jiang, D.; Zhang, Y.; Dubonos, S. V.; Grigorieva, I. V.; Firsov, A. A. Electric Field Effect in Atomically Thin Carbon Films. Science 2004, 306, 666-669.

(2) Banszerus, L.; Schmitz, M.; Engels, S.; Dauber, J.; Oellers, M.; Haupt, F.; Watanabe, K.; Taniguchi, T.; Beschoten, B.; Stampfer, C. Ultrahigh-mobility graphene devices from chemical vapor deposition on reusable copper. Sci. Adv. 2015, 1, No. e1500222. 
(3) Heiranian, M.; Farimani, A. B.; Aluru, N. R. Water desalination with a single-layer MoS2 nanopore. Nat. Commun. 2015, 6, 8616.

(4) Liu, K.; Feng, J.; Kis, A.; Radenovic, A. Atomically Thin Molybdenum Disulfide Nanopores with High Sensitivity for DNA Translocation. ACS Nano 2014, 8, 2504-2511.

(5) Weng, Q.; Wang, X.; Wang, X.; Bando, Y.; Golberg, D. Functionalized hexagonal boron nitride nanomaterials: emerging properties and applications. Chem. Soc. Rev. 2016, 45, 3989-4012.

(6) Xiao, F.; Naficy, S.; Casillas, G.; Khan, M. H.; Katkus, T.; Jiang, L.; Liu, H.; Li, H.; Huang, Z. Edge-Hydroxylated Boron Nitride Nanosheets as an Effective Additive to Improve the Thermal Response of Hydrogels. Adv. Mater. 2015, 27, 7196-7203.

(7) Lei, W.; Mochalin, V. N.; Liu, D.; Qin, S.; Gogotsi, Y.; Chen, Y. Boron nitride colloidal solutions, ultralight aerogels and freestanding membranes through one-step exfoliation and functionalization. Nat. Commun. 2015, 6, 8849.

(8) Sainsbury, T.; Satti, A.; May, P.; Wang, Z.; McGovern, I.; Gun'ko, Y. K.; Coleman, J. Oxygen Radical Functionalization of Boron Nitride Nanosheets. J. Am. Chem. Soc. 2012, 134, 1875818771.

(9) Sainsbury, T.; O’Neill, A.; Passarelli, M. K.; Seraffon, M.; Gohil, D.; Gnaniah, S.; Spencer, S. J.; Rae, A.; Coleman, J. N. Dibromocarbene Functionalization of Boron Nitride Nanosheets: Toward Band Gap Manipulation and Nanocomposite Applications. Chem. Mater. 2014, 26, 7039-7050.

(10) Shin, H.; Guan, J.; Zgierski, M. Z.; Kim, K. S.; Kingston, C. T.; Simard, B. Covalent Functionalization of Boron Nitride Nanotubes via Reduction Chemistry. ACS Nano 2015, 9, 12573-12582.

(11) Aurbach, D.; Gottlieb, H. The electrochemical behavior of selected polar aprotic systems. Electrochim. Acta 1989, 34, 141-156.

(12) Bacle, P.; Seitsonen, A. P.; Iannuzzi, M.; Hutter, J. Chemical Reactions on Metalsupported Hexagonal Boron Nitride Investigated with Density Functional Theory. Chimia 2014, 68, 596-601.

(13) Mousavi, M. P. S.; Kashefolgheta, S.; Stein, A.; Bühlmann, P. Electrochemical Stability of Quaternary Ammonium Cations: An Experimental and Computational Study. J. Electrochem. Soc. 2016, 163, H74-H80.

(14) Bjerglund, E.; Kongsfelt, M.; Shimizu, K.; Jensen, B. B. E.; Koefoed, L.; Ceccato, M.; Skrydstrup, T.; Pedersen, S. U.; Daasbjerg, K. Controlled Electrochemical Carboxylation of Graphene To Create a Versatile Chemical Platform for Further Functionalization. Langmuir 2014, 30, 6622-6628.

(15) Koefoed, L.; Kongsfelt, M.; Ulstrup, S.; Cabo, A. G.; Cassidy, A.; Whelan, P. R.; Bianchi, M.; Dendzik, M.; Pizzocchero, F.; Jørgensen, B.; Bøggild, P.; Hornekaer, L.; Hofmann, P.; Pedersen, S. U.; Daasbjerg, K. Facile electrochemical transfer of largearea single crystal epitaxial graphene from $\operatorname{Ir}(111)$. J. Phys. D: Appl. Phys. 2015, $48,115306$.

(16) Salmi, Z.; Koefoed, L.; Jensen, B.; Cabo, A. G.; Hofmann, P.; Pedersen, S. U.; Daasbjerg, K. Electroinduced intercalation of tetraalkylammonium ions at the interface of graphene grown on copper, platinum, and iridium. ChemElectroChem 2016, 3, 22022211.

(17) Miniussi, E.; Bernard, C.; Cun, H. Y.; Probst, B.; Leuenberger, D.; Mette, G.; Zabka, W.-D.; Weinl, M.; Haluska, M.; Schreck, M.; Osterwalder, J.; Greber, T. Fermi surface map of large-scale singleorientation graphene on $\mathrm{SiO}$ 2. J. Phys.: Condens. Matter 2017, 29, 475001.

(18) Cun, H.; Hemmi, A.; Miniussi, E.; Bernard, C.; Probst, B.; Liu, K.; Alexander, D. T. L.; Kleibert, A.; Mette, G.; Weinl, M.; Schreck, M.; Osterwalder, J.; Radenovic, A.; Greber, T. Centimeter-Sized Single-Orientation Monolayer Hexagonal Boron Nitride With or Without Nanovoids. Nano Lett. 2018, 18, 1205-1212.

(19) Chen, M.-W.; Kim, H.; Bernard, C.; Pizzochero, M.; Zaldívar, J.; Pascual, J. I.; Ugeda, M. M.; Yazyev, O. V.; Greber, T.; Osterwalder, J.; Renault, O.; Kis, A. Electronic Properties of Transferable Atomically Thin MoSe2/h-BN Heterostructures Grown on $\mathrm{Rh}(111)$. ACS Nano 2018, 12, 11161-11168.
(20) Mayell, J. S.; Bard, A. J. The Electroreduction of Quaternary Ammonium Compounds. J. Am. Chem. Soc. 1963, 85, 421-425.

(21) Kroon, M. C.; Buijs, W.; Peters, C. J.; Witkamp, G.-J. Decomposition of ionic liquids in electrochemical processing. Green Chem. 2006, 8, 241-245.

(22) Preobrajenski, A. B.; Vinogradov, A. S.; Ng, M. L.; Ćavar, E.; Westerström, R.; Mikkelsen, A.; Lundgren, E.; Mårtensson, N. Influence of chemical interaction at the lattice-mismatched $h$-BN/ $\mathrm{Rh}(111)$ and $h-\mathrm{BN} / \mathrm{Pt}(111)$ interfaces on the overlayer morphology. Phys. Rev. B: Condens. Matter Mater. Phys. 2007, 75, 245412.

(23) Corso, M.; Auwärter, W.; Muntwiler, M.; Tamai, A.; Greber, T.; Osterwalder, J. Boron Nitride Nanomesh. Science 2004, 303, 217220.

(24) Sugiyama, Y.; Bernard, C.; Okuyama, Y.; Ideta, S.; Tanaka, K.; Greber, T.; Hirahara, T. Flattening and manipulation of the electronic structure of h-BN/Rh(111) nanomesh upon Sn intercalation. Surf. Sci. 2018, 672-673, 33-38.

(25) Brugger, T.; Ma, H.; Iannuzzi, M.; Berner, S.; Winkler, A.; Hutter, J.; Osterwalder, J.; Greber, T. Nanotexture Switching of Single-Layer Hexagonal Boron Nitride on Rhodium by Intercalation of Hydrogen Atoms. Angew. Chem., Int. Ed. 2010, 49, 6120-6124.

(26) Slater, J. C.; Johnson, K. H. Self-Consistent-Field X $\alpha$ Cluster Method for Polyatomic Molecules and Solids. Phys. Rev. B 1972, 5, 844-853.

(27) Hemmi, A.; Bernard, C.; Cun, H.; Roth, S.; Klöckner, M.; Kälin, T.; Weinl, M.; Gsell, S.; Schreck, M.; Osterwalder, J.; Greber, T. High quality single atomic layer deposition of hexagonal boron nitride on single crystalline $\mathrm{Rh}(111)$ four-inch wafers. Rev. Sci. Instrum. 2014, 85, No. 035101.

(28) Goriachko, A.; He; Knapp, M.; Over, H.; Corso, M.; Brugger, T.; Berner, S.; Osterwalder, J.; Greber, T. Self-Assembly of a Hexagonal Boron Nitride Nanomesh on $\mathrm{Ru}(0001)$. Langmuir 2007, 23, 2928-2931.

(29) Sutter, P.; Cortes, R.; Lahiri, J.; Sutter, E. Interface Formation in Monolayer Graphene-Boron Nitride Heterostructures. Nano Lett. 2012, 12, 4869-4874.

(30) Greber, T.; Raetzo, O.; Kreutz, T. J.; Schwaller, P.; Deichmann, W.; Wetli, E.; Osterwalder, J. A photoelectron spectrometer for kspace mapping above the Fermi level. Rev. Sci. Instrum. 1997, 68, 4549-4554.

(31) Muntwiler, M.; Zhang, J.; Stania, R.; Matsui, F.; Oberta, P.; Flechsig, U.; Patthey, L.; Quitmann, C.; Glatzel, T.; Widmer, R.; Meyer, E.; Jung, T. A.; Aebi, P.; Fasel, R.; Greber, T. Surface science at the PEARL beamline of the Swiss Light Source. J. Synchrotron Radiat. 2017, 24, 354-366.

(32) Hutter, J.; Iannuzzi, M.; Schiffmann, F.; VandeVondele, J. cp2k: atomistic simulations of condensed matter systems. Wiley Interdiscip. Rev. Comput. Mol. Sci. 2014, 4, 15-25.

(33) Perdew, J. P.; Burke, K.; Ernzerhof, M. Generalized Gradient Approximation Made Simple. Phys. Rev. Lett. 1996, 77, 3865-3868.

(34) Vydrov, O. A.; Van Voorhis, T. Nonlocal van der Waals Density Functional Made Simple. Phys. Rev. Lett. 2009, 103, 63004-63007.

(35) Sabatini, R.; Gorni, T.; de Gironcoli, S. Nonlocal van der Waals density functional made simple and efficient. Phys. Rev. B: Condens. Matter Mater. Phys. 2013, 87, 41108-41111.

(36) Goedecker, S.; Teter, M.; Hutter, J. Separable dual-space Gaussian pseudopotentials. Phys. Rev. B: Condens. Matter Mater. Phys. 1996, 54, 1703-1710.

(37) VandeVondele, J.; Hutter, J. Gaussian basis sets for accurate calculations on molecular systems in gas and condensed phases. $J$. Chem. Phys. 2007, 127, 114105.

(38) Iannuzzi, M.; Hutter, J. Inner-shell spectroscopy by the Gaussian and augmented plane wave method. Phys. Chem. Chem. Phys. 2007, 9, 1599-1610. 